\title{
Epidemiological Distribution and Subtype Analysis of Premenstrual Dysphoric Disorder Syndromes and Symptoms Based on TCM Theories
}

\author{
Mingqi Qiao,, ${ }^{1}$ Peng Sun, ${ }_{1}^{1}$ Haijun Wang, ${ }^{2}$ Yang Wang, ${ }^{3}$ Xianghong Zhan, ${ }^{4}$ Hongqi Liu, ${ }^{5}$ \\ Xiaoyun Wang, ${ }^{6}$ Xia Li, ${ }^{7}$ Xiaoru Wang, ${ }^{7}$ Jibiao Wu, ${ }^{1}$ and Fushun Wang ${ }^{8}$ \\ ${ }^{1}$ Key Laboratory of Traditional Chinese Medicine for Classical Theory, Ministry of Education, \\ Shandong University of Traditional Chinese Medicine, Jinan, Shandong Province, China \\ ${ }^{2}$ National Key Subject of TCM Psychology, State Administration of Traditional Chinese Medicine, Jinan, Shandong Province, China \\ ${ }^{3}$ Laboratory of Ethnopharmacology, Institute of Integrated Traditional Chinese and Western Medicine, Xiangya Hospital, \\ Central South University, Changsha, Hunan Province, China \\ ${ }^{4}$ School of Preclinical Medical Sciences, Henan University of Traditional Chinese Medicine, Zhengzhou, Henan Province, China \\ ${ }^{5}$ The Affiliated Hospital of Shanxi University of Traditional Chinese Medicine, Taiyuan, Shanxi Province, China \\ ${ }^{6}$ The Second Affiliated Hospital of Guangzhou University of Chinese Medicine, Guangzhou, Guangdong Province, China \\ ${ }^{7}$ The Central Hospital of Jinan City, Jinan, Shandong Province, China \\ ${ }^{8}$ Department of Psychology, Nanjing University of Chinese Medicine, Nanjing, Jiangsu Province, China
}

Correspondence should be addressed to Peng Sun; sunpeng369@126.com

Received 28 November 2016; Revised 6 May 2017; Accepted 23 May 2017; Published 15 June 2017

Academic Editor: Adair Santos

Copyright (c) 2017 Mingqi Qiao et al. This is an open access article distributed under the Creative Commons Attribution License, which permits unrestricted use, distribution, and reproduction in any medium, provided the original work is properly cited.

\begin{abstract}
We performed an epidemiological investigation of subjects with premenstrual dysphoric disorder (PMDD) to identify the clinical distribution of the major syndromes and symptoms. The pathogenesis of PMDD mainly involves the dysfunction of liver conveyance and dispersion. Excessive liver conveyance and dispersion are associated with liver-qi invasion syndrome, while insufficient liver conveyance and dispersion are expressed as liver-qi depression syndrome. Additionally, a nonconditional logistic regression was performed to analyze the symptomatic features of liver-qi invasion and liver-qi depression. As a result of this analysis, two subtypes of PMDD are proposed, namely, excessive liver conveyance and dispersion (liver-qi invasion syndrome) and insufficient liver conveyance and dispersion (liver-qi depression syndrome). Our findings provide an epidemiological foundation for the clinical diagnosis and treatment of PMDD based on the identification of different types.
\end{abstract}

\section{Introduction}

With a prevalence rate of $3 \%-8 \%[1-3]$, premenstrual dysphoric disorder (PMDD) is characterized by a group of symptoms that manifest as emotional disorders and painful breast distension. PMDD can significantly affect one's work and social functioning one week before the menstrual period. Different subtypes of PMDD have been described [4-8], and selecting the appropriate treatment for the subtype is an important consideration for management. As early as 1983 , it was suggested that premenstrual syndrome (PMS) could be divided into four subtypes, namely, PMS-A (anxiety), PMS-D (depression), PMS-C (cravings), and PMS-H (hyperhydration) [9]. Subsequently, a large number of clinical studies demonstrate that fluoxetine, the first-line treatment for PMDD, can address some of the symptoms of PMDD, including dysphoria [10], food cravings [11], tension, and anxiety [12-14], but it has no effect on other clinical manifestations, such as sexual hypoactivity, indicating that PMDD has subtypes that differ in their pathogenesis. The effective rate of fluoxetine treatment is less than 60\% [15], which may be due to the existence of different central mechanisms for the different PMDD subtypes. Through epidemiological investigations in China [16] it has been shown that the four syndromes of liver-qi invasion, liver-qi depression, liver fire 
flaring, and heart-spleen deficiency account for 95\% of the symptoms of PMDD (58.9\% and 27.5\% for liver-qi invasion and liver-qi depression, resp.), and appropriate treatments, such as Jingqianping granules and Jingqianshu granules, have been developed.

Based on the findings described above, the existence of PMDD subtypes has been recognized by the medical community both in China and abroad, but the classification systems and treatment goals vary. This research was conducted as an epidemiological investigation of the relationship between TCM syndromes and the symptoms of PMDD. Subtypes of PMDD are described based on the pathogenic mechanisms addressed in TCM and on the symptoms reported by the patients.

\section{Materials and Methods}

2.1. Ethics Statement. This study was approved by Shandong University of Traditional Chinese Medicine and the State Administration of Traditional Chinese Medicine. The patients and healthy volunteers who participated in this study all signed informed consent forms.

2.2. Inclusion and Exclusion Criteria. Inclusion criteria included symptoms consistent with PMDD as described in the Diagnostic and Statistical Manual of Mental Disorders (DSM-IV); women ranging in age from 18 to 45 years, with no exclusion based on race; possessing clear consciousness and independence as well as active judgment; being able to understand the research goals and willing to cooperate; no severe disease of the heart, liver, or kidney, no brain tumor or other craniocerebral diseases, and no history of taking psychotropic drugs.

The diagnostic criteria for liver-qi invasion and liver-qi depression are based on the results of previous epidemiological research, "The 7th Edition of Basic Theory of TCM," and the Delphi method. The diagnostic criteria for the diagnoses of liver depression forming fire, liver stagnation and spleen deficiency, liver depression and blood stasis, liver depression and kidney deficiency, and spleen and kidney deficiency syndromes are based on "Diagnostics of TCM," "Guiding Principles of Clinical Research on TCM New Drugs," and the Delphi method. The diagnostic criteria for liver-qi invasion and liver-qi depression syndromes are described below.

Diagnostic criteria for liver-qi invasion syndrome are as follows: major symptoms: premenstrual dysphoria and irritability; painful distension of the breasts; decreased function in work, household management, and social activity; minor symptoms: distension in the head, headache, chest distress, anxiety, dreaminess, constipation, and premature menstruation; tongue vein: light red or red tongue, white or slightly yellow coating, and a weak pulse. Diagnosis: the diagnosis of liver-qi invasion is confirmed if two of the major symptoms and three or more of the minor symptoms are present in combination with the characteristics of the tongue vein.

Diagnostic criteria for liver-qi depression syndrome are as follows: major symptoms: low spirits or depression and anger during the premenstrual phase; chest distress; decreased function in work, household management, and social activity; minor symptoms: painful distension of the breasts, chestcoerced distending pain, preference for sigh, abdominal bulge, anorexia, loss of strength, dysmenorrhea, reduced discharge during menses, and delayed menstruation; tongue vein: slightly dark or dark tongue, fat tongue body, slightly white or yellow coating, and a weak or slow pulse. Diagnosis: the diagnosis is confirmed if two of the major symptoms (poor mood or depression and anger during the premenstrual phase is required) and three of the minor symptoms are present in combination with the characteristics of the tongue vein.

Exclusion criteria are as follows: psychiatric patients; patients with severe physical diseases; a history of drug abuse (including the use of drugs to treat PMDD within three months); hematological diseases; pregnancy or lactation; patients who are unable to cooperate during visits due to aphasia, a disturbance of consciousness, dementia, or other conditions; patients with chronic five-organ diseases and clinical symptoms; patients who had undergone unilateral oophorectomy or abortion within six months.

2.3. Patient Demographics. An investigation of 3,530 cases of child-bearing women from Shandong, Henan, Guangzhou, and Shanxi provinces was conducted. In total, 675 cases were selected for observation, including 107 cases from Shandong Traditional Chinese Medicine University, 250 cases from Henan College of Traditional Chinese Medicine and its affiliated hospital, 149 cases from The Second Affiliated Hospital of Guangzhou University of Traditional Chinese Medicine, and 169 cases from the Affiliated Hospital of Shanxi College of Traditional Chinese Medicine.

Ethnicity: the cases included 662 Han Chinese (98.1\%) and 13 individuals of other ethnicities (1.9\%); age: 521 subjects were aged $18-28(77.2 \%), 118$ subjects were aged $29-39(17.5 \%)$, and 31 subjects were aged 40-48 (4.6\%); marriage status: 513 subjects were unmarried; 162 were married; profession: 391 subjects were students (57.9\%), 91 were office clerks (13.5\%), 33 were self-employed (4.9\%), 34 were doctors $(5.0 \%), 10$ were teachers $(1.5 \%), 14$ were civil servants $(2.1 \%), 30$ were unemployed $(4.5 \%), 8$ were laborers $(1.2 \%), 2$ were farmers $(0.3 \%)$, and the profession was unknown for 62 subjects (9.2\%); educational level: 496 subjects had a college education (73.5\%), 96 had a postgraduate education (14.2\%), 61 had a high school (technical secondary school) education (9.0\%), 15 had a middle school education (2.2\%), and 7 had a primary school education or below (1.0\%).

2.4. Methodology. This study was designed as a crosssectional epidemiological investigation [17-19]. The survey included items related to the participants' general information and medical history, the Information Collection Table of PMDD TCM Symptoms, information for TCM syndrome differentiation (based on expert experience), and a daily record of the severity of problems (DRSP). Frequency analysis and nonconditional logistic regression analysis $[20,21]$ were performed to evaluate the PMDD subtypes in combination with the pathogenesis and symptoms according to TCM. 


\section{Results}

3.1. Distribution of PMDD Syndromes (675 Cases). Through a cross-sectional epidemiological analysis of the 675 patients with PMDD, a frequency analysis was performed to summarize the distribution of PMDD syndromes, as shown in Table 1 .

The major syndromes refer to the primary syndromes of the disease. A patient will present a specific syndrome; for example, liver-qi depression is a major syndrome of PMDD. Minor syndromes indicate the presence of the major syndrome of the disease, and the patient may also present another syndrome with a relatively complete manifestation. For example, liver-qi depression syndrome, a major syndrome of PMDD, is accompanied by liver and kidney deficiency syndrome. The accompanying syndromes are expressions or symptoms of other syndromes associated with the disease. The key difference between the accompanying syndromes and the minor syndromes mainly lies in whether a complete manifestation of the syndrome is present.

From Table 1, it can be seen that the major syndromes of PMDD include liver-qi invasion and liver-qi depression. Liver stagnation and spleen deficiency, liver depression forming fire and liver depression, and blood stasis are also common. The syndromes of liver depression and kidney deficiency, as well as spleen and kidney deficiency, were present at relatively low frequencies, but they tended to emerge as nonmajor syndromes. The pathogenesis of most of these syndromes is related to the liver, with the dysfunction of liver conveyance and dispersion playing the most important role.

3.2. Distribution of PMDD Symptoms (675 Cases). Through a cross-sectional epidemiological analysis of the 675 patients with PMDD, a frequency analysis was conducted to summarize the distribution of the PMDD symptoms, as shown in Table 2.

From Table 2, it can be seen that there are three types of PMDD symptoms, namely, emotional symptoms, physical symptoms, and symptoms related to social function. Major symptoms include the following: emotional symptoms (impatience and irritability, emotional depression, anxiety, low spirits, and hopeless feelings) and physical symptoms (fatigue, cold intolerance and cold limbs, insomnia, dreaminess, head distension, headache, dizziness, dry mouth and throat, chest distress, chest-coerced distension, painful distension of the breasts, preference for sighing, anorexia, abdominal bulge, painful distension of the abdomen, soreness and weakness of the waist and knees, loose stool, sexual hypoactivity, dysmenorrhea, reduced discharge during menses and dark purple menstrual flow, and impaired social function affecting the ability to work, manage household tasks, and learn).

The typical clinical manifestation of PMDD is dominated by emotional symptoms, such as impatience and irritability, as well as emotional depression. Impatience and irritability are typical symptoms of excessive liver conveyance and dispersion and emotional depression is associated with insufficient liver conveyance and dispersion. The liver-qi invasion and liver-qi depression syndromes play a dominant role in the PMDD syndromes. Therefore, analyzing the symptomatic features of the liver-qi invasion and liver-qi depression syndromes is important for establishing the PMDD subtype.

3.3. Analysis of the Symptomatic Features of PMDD Liver-Qi Invasion Syndrome. With the presence or absence of liver-qi invasion syndrome as the dependent variable and the relevant symptoms as independent variables, a nonconditional logistic regression analysis was performed to analyze the symptomatic features of PMDD liver-qi invasion syndrome, as shown in Table 3.

The results show that impatience and irritability, head distension, headache, painful distension of the breasts, abdominal bulge, and loose stool tended to emerge as a group of internally related symptoms, demonstrating the pathogenic features of qi abnormal rising for excessive liver conveyance and dispersion.

3.4. Analysis of the Symptomatic Features of PMDD LiverQi Depression Syndrome. With the presence or absence of liver-qi depression as the dependent variable and the relevant symptoms as the independent variables, a nonconditional logistic regression analysis was performed to analyze the symptomatic features of PMDD liver-qi depression syndrome, as shown in Table 4.

The results show that emotional depression, painful distension of the breasts, and reduced discharge during menses represent a group of internally related symptoms, demonstrating the pathogenic features of qi stagnation due to insufficient liver-qi conveyance and dispersion.

\section{Discussion}

To improve readers' ability to understand this research, efforts have been made to use English terminology to discuss the concepts and words used in traditional Chinese medicine (TCM). Although some of the concepts in TCM have no corresponding words in English and there is no standard reference for the translation of certain expressions, we hope that our interpretations (Table 5) will aid in the understanding of the theories that underlie TCM. The concepts used in TCM have been shown to be effective and can guide treatment decisions in the clinic; nonetheless, TCM remains marginalized in current medical practice. The explanations of relevant concepts and expressions do not comprehensively describe TCM theory, but they are intended to provide readers with a better understanding of the research content and original results described in this paper.

Based on the studies described above, the pathogenic mechanism of PMDD is mainly related to the disorder of liver conveyance and dispersion, thus involving the spleen and kidneys. The pathogenic site mainly involves the circulating parts of the jueyin liver meridian of the foot; therefore, differentiation is based on the liver. The liver controls conveyance and dispersion, and liver dysfunction can cause excessive or insufficient conveyance and dispersion. Symptoms such as impatience and irritability accompanied by head distension, 


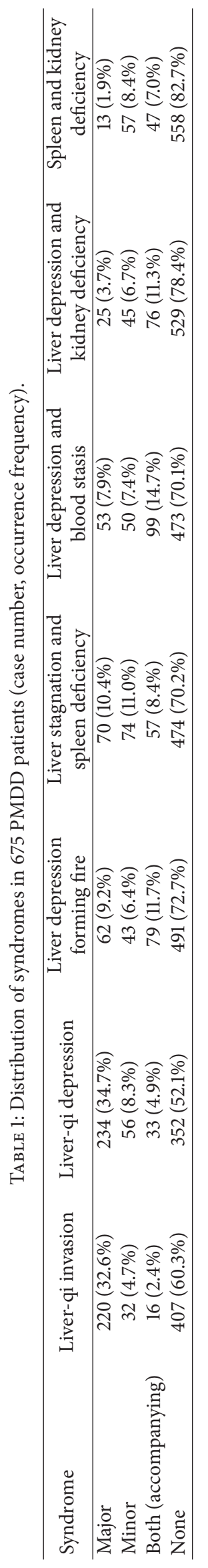




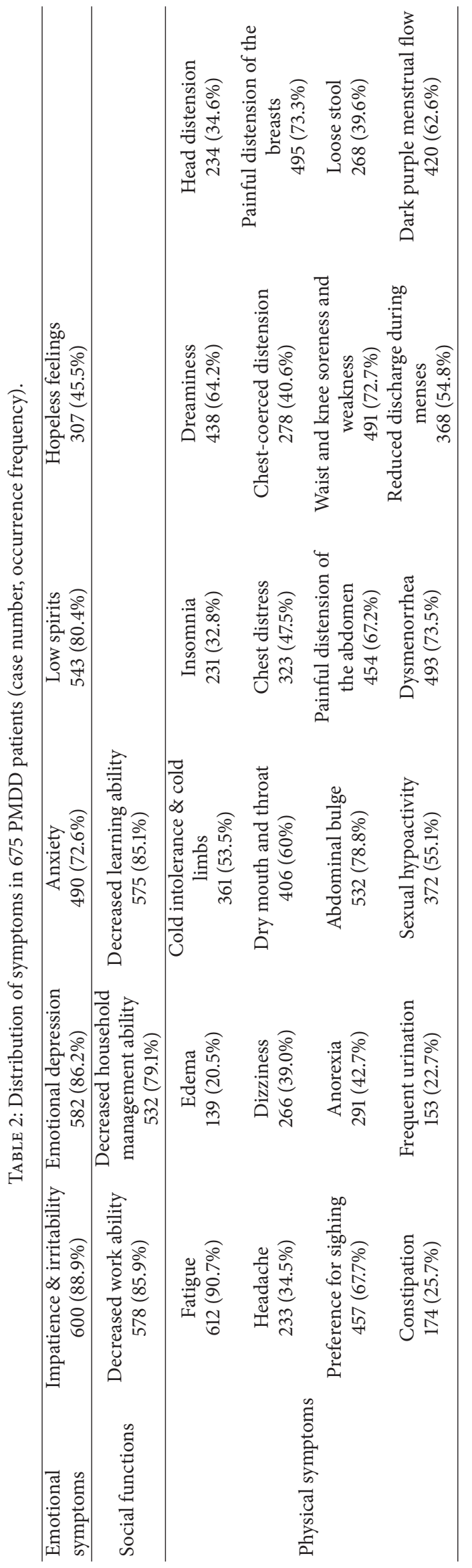


TABLE 3: Results of a nonconditional logistic regression analysis of the symptomatic features of liver-qi invasion syndrome.

\begin{tabular}{|c|c|c|c|c|c|}
\hline & $B$ & SE & Wald & Sig. & $\operatorname{Exp}(B)$ \\
\hline Impatience \& irritability & 0.985 & 0.141 & 49.005 & 0.000 & 2.677 \\
\hline Fatigue & -0.058 & 0.137 & 0.181 & 0.670 & 0.943 \\
\hline Head distension & 0.135 & 0.172 & 0.615 & 0.433 & 1.144 \\
\hline Headache & 0.075 & 0.141 & 0.278 & 0.598 & 1.077 \\
\hline Dizziness & 0.408 & 0.150 & 7.436 & 0.006 & 1.504 \\
\hline Painful distension of the breasts & 0.054 & 0.122 & 0.199 & 0.656 & 1.056 \\
\hline Chest-coerced distension & -0.028 & 0.189 & 0.023 & 0.881 & 0.972 \\
\hline Chest distress & 0.095 & 0.126 & 0.575 & 0.448 & 1.100 \\
\hline Preference for sighing & -0.254 & 1.120 & 4.481 & 0.034 & 0.775 \\
\hline Abdominal bulge & 0.030 & 0.125 & 0.059 & 0.808 & 1.031 \\
\hline Waist and knee soreness and weakness & -0.214 & 0.111 & 3.728 & 0.053 & 0.807 \\
\hline Loose stool & 0.021 & 0.119 & 0.030 & 0.862 & 1.021 \\
\hline Constant & -3.005 & 0.489 & 37.757 & 0.000 & 0.050 \\
\hline
\end{tabular}

TABLE 4: Results of a nonconditional logistic regression analysis of the symptomatic features of liver-qi depression syndrome.

\begin{tabular}{|c|c|c|c|c|c|}
\hline & $B$ & SE & Wald & Sig. & $\operatorname{Exp}(B)$ \\
\hline Emotional depression & 0.476 & 0.117 & 16.427 & 0.000 & 1.610 \\
\hline Fatigue & -0.166 & 0.129 & 1.647 & 0.199 & 0.847 \\
\hline Chest distress & -0.116 & 0.120 & 0.949 & 0.330 & 0.890 \\
\hline Chest-coerced distension & -0.030 & 0.178 & 0.028 & 0.868 & 0.971 \\
\hline Painful distension of the breasts & 0.076 & 0.118 & 0.415 & 0.519 & 1.079 \\
\hline Preference for sighing & -0.114 & 0.111 & 1.041 & 0.308 & 0.893 \\
\hline Anorexia & -0.096 & 0.112 & 0.728 & 0.394 & 0.909 \\
\hline Abdominal bulge & -0.103 & 0.118 & 0.772 & 0.380 & 0.902 \\
\hline Waist and knees soreness and weakness & -0.176 & 0.103 & 2.882 & 0.090 & 0.839 \\
\hline Dysmenorrhea & -0.023 & 0.192 & 0.014 & 0.906 & 0.978 \\
\hline Reduced discharge during menses & 0.134 & 0.180 & 0.551 & 0.458 & 1.143 \\
\hline Dark purple menstrual flow & -0.162 & 0.177 & 0.837 & 0.360 & 0.851 \\
\hline Sexual hypoactivity & -0.071 & 0.106 & 0.447 & 0.504 & 0.931 \\
\hline Constant & 0.427 & 0.550 & 0.603 & 0.437 & 1.532 \\
\hline
\end{tabular}

headache, dizziness, painful distension of the breasts, abdominal bloating, loose stool, and others are clinical manifestation of excessive conveyance and dispersion. Insufficient conveyance and dispersion are characterized by emotional depression and low spirits accompanied by painful distension of the breasts and reduced discharge during menses. Apart from the occurrence of painful distension of the breasts, which occurs in both excessive and insufficient conveyance and dispersion, the symptoms listed above can differentiate the pathogenesis of PMDD, demonstrating that there are at least two relatively independent clinical subtypes, namely, liver-qi invasion and liver-qi depression syndromes. Because other syndromes, such as liver depression forming fire, liver stagnation and spleen deficiency, liver depression and blood stasis, and liver depression and kidney deficiency, are components of liver-qi invasion and liver-qi depression syndromes and are less distributed among patients; they lack discriminatory power. This research confirms that there are at least two primary subtypes of PMDD, namely, liver-qi invasion and liver-qi depression syndromes.

Liver-qi invasion syndrome indicates excessive qi conveyance and dispersion as well as qi and blood rising; therefore, the jueyin liver meridian of the foot prompts impatience and irritability, head distension, headache, dizziness, and chest distress. Liver-qi depression indicates insufficient qi conveyance and dispersion; therefore, emotional depression, low spirits, no liver upbearing and effusion, unsmooth qi flow, and reduced discharge during menses are reported. Painful distension of the breasts is a common manifestation of disturbances of qi movement in the liver meridian, demonstrating that the two subtypes (syndromes) have a common mechanism but different features. Compared with the subtype classifications used throughout the world, the PMDD subtypes described in this paper are characterized by obvious features that are differentiated according to TCM and provide valuable clinical guidance. 


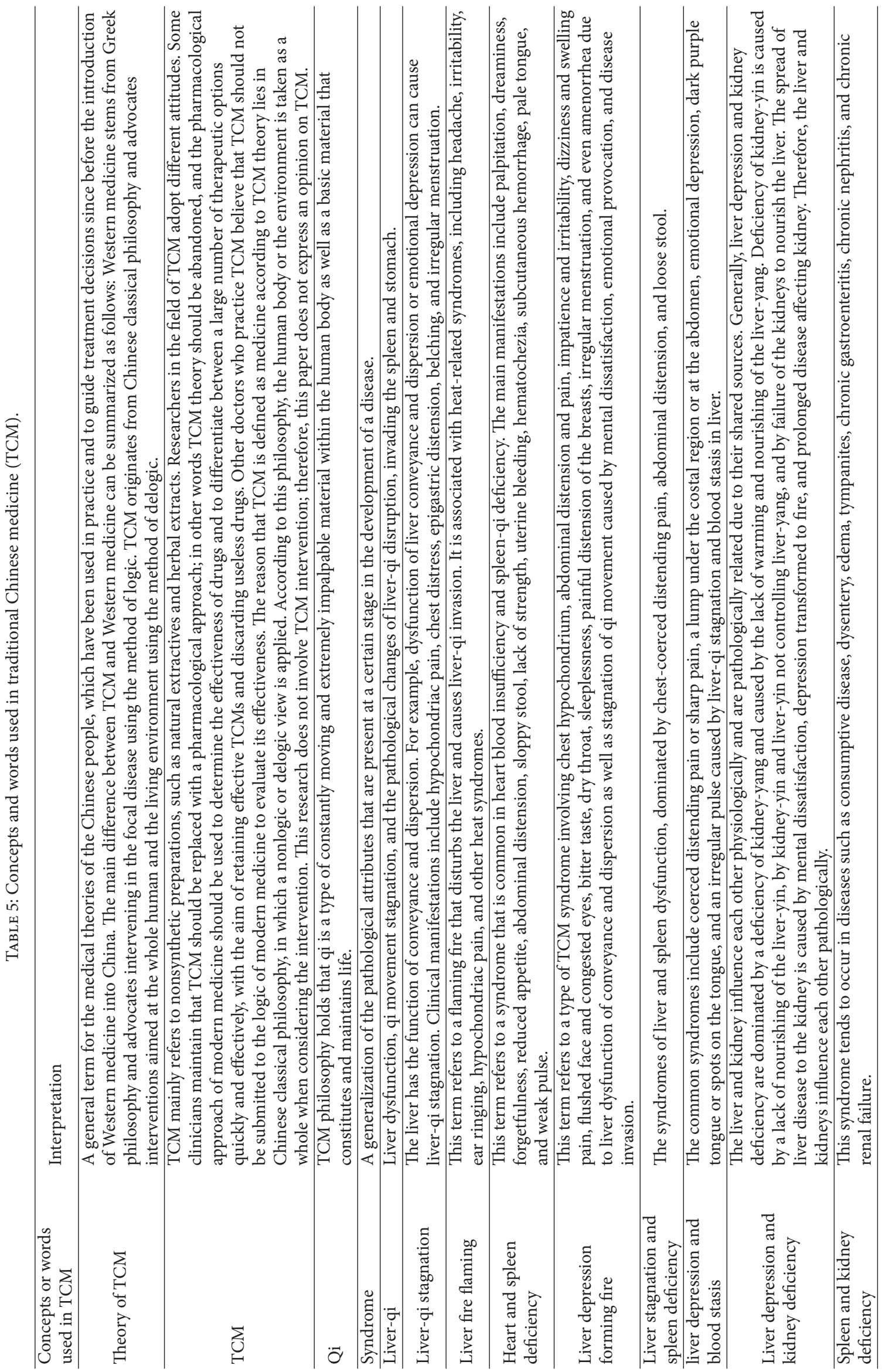




\section{Conclusion}

Liver dysfunction is the major pathogenic feature used in the differentiation of the clinical syndromes of PMDD. Excessive and insufficient liver conveyance and dispersion can cause two types of clinical manifestations, pointing to the existence of independent subtypes of PMDD. Suppressing a hyperactive liver to address descending adverse qi and discharging the liver to relieve depression can provide improved clinical results.

\section{Ethical Approval}

The study design was approved by the appropriate ethics review boards.

\section{Consent}

All study participants provided informed consent.

\section{Conflicts of Interest}

There are no conflicts of interest to declare.

\section{Authors' Contributions}

All authors discussed the results and approved the manuscript.

\section{Acknowledgments}

This work was supported by Special Funds for Major State Basic Research Programme (2011CB505102).

\section{References}

[1] S. Gehlert and S. Hartlage, "A design for studying the DSM-IV research criteria of premenstrual dysphoric disorder," Journal of Psychosomatic Obstetrics \& Gynecology, vol. 18, no. 1, pp. 36-44, 2009.

[2] F. E. Omu, R. Al-Marzouk, H. Delles, N. O. Oranye, and A. E. Omu, "Premenstrual dysphoric disorder: prevalence and effects on nursing students' academic performance and clinical training in Kuwait," Journal of Clinical Nursing, vol. 20, no. 1920, pp. 2915-2923, 2011.

[3] G. V. Wallenstein, B. Blaisdell-Gross, K. Gajria et al., "Development and validation of the premenstrual symptoms impact survey (PMSIS): A disease-specific quality of life assessment tool," Journal of Women's Health, vol. 17, no. 3, pp. 439-450, 2008.

[4] A. P. Borrow and N. M. Cameron, "Estrogenic mediation of serotonergic and neurotrophic systems: implications for female mood disorders," Progress in Neuro-Psychopharmacology and Biological Psychiatry, vol. 54, pp. 13-25, 2014.

[5] E. W. Freeman, M. D. Sammel, H. Lin, K. Rickels, and S. J. Sondheimer, "Clinical subtypes of premenstrual syndrome and responses to sertraline treatment," Obstetrics and Gynecology, vol. 118, no. 6, pp. 1293-1300, 2011.
[6] J. Griffiths and T. Lovick, "Withdrawal from progesterone increases expression of alpha4, betal, and delta GABA(A) receptor subunits in neurons in the periaqueductal gray matter in female wistar rats," Journal of Comparative Neurology, vol. 486, no. 1, pp. 89-97, 2005.

[7] Y. Inoue, T. Terao, N. Iwata et al., "Fluctuating serotonergic function in premenstrual dysphoric disorder and premenstrual syndrome: findings from neuroendocrine challenge tests," Psychopharmacology, vol. 190, no. 2, pp. 213-219, 2007.

[8] K. M. Ismail, T. Nevatte, S. O'Brien et al., "Clinical subtypes of core premenstrual disorders: a delphi survey," Archives of Women's Mental Health, vol. 16, no. 3, pp. 197-201, 2013.

[9] G. E. Abraham, "Nutritional factors in the etiology of the premenstrual tension syndromes," The Journal of Reproductive Medicine, vol. 28, no. 7, pp. 446-464, 1981.

[10] Y. Li, A. L. Pehrson, D. P. Budac, C. Sánchez, and M. Gulinello, "A rodent model of premenstrual dysphoria: progesterone withdrawal induces depression-like behavior that is differentially sensitive to classes of antidepressants," Behavioural Brain Research, vol. 234, no. 2, pp. 238-247, 2012.

[11] D. J. Anderson, N. J. Legg, and D. A. Ridout, "Preliminary trial of photic stimulation for premenstrual syndrome," Journal of Obstetrics and Gynaecology, vol. 17, no. 1, pp. 76-79, 1997.

[12] E. M. Steinberg, G. M. P. Cardoso, P. E. Martinez, D. R. Rubinow, and P. J. Schmidt, "Rapid response to fluoxetine in women with premenstrual dysphoric disorder," Depression and Anxiety, vol. 29, no. 6, pp. 531-540, 2012.

[13] M. Steiner, S. Steinberg, D. Stewart et al., "Fluoxetine in the treatment of premenstrual dysphoria," New England Journal of Medicine, vol. 332, no. 23, pp. 1529-1534, 1995.

[14] S. H. Wood, J. F. Mortola, Y. F. Chan, F. Moossazadeh, and S. S. Yen, "Treatment of premenstrual syndrome with fluoxetine: a double-blind, placebo-controlled, crossover study," Obstetrics and Gynecology, vol. 80, no. 3, pp. 339-344, 1992.

[15] U. Halbreich, P. M. S. O’Brien, E. Eriksson, T. Bäckström, K. A. Yonkers, and E. W. Freeman, "Are there differential symptom profiles that improve in response to different pharmacological treatments of premenstrual syndrome/premenstrual dysphoric disorder?" CNS Drugs, vol. 20, no. 7, pp. 523-547, 2006.

[16] M. Qiao, Z. Zhang, X. Xu et al., "Epidemiological survey on syndromes distribution in PMS," Journal of Basic Medicine in Traditional Chinese Medicine, no. 3, pp. 31-33, 1997.

[17] A. Anastassaki Köhler, A. Hugoson, and T. Magnusson, "Prevalence of symptoms indicative of temporomandibular disorders in adults: cross-sectional epidemiological investigations covering two decades," Acta Odontologica Scandinavica, vol. 70, no. 3, pp. 213-223, 2012.

[18] N. U. Odo, J. H. Mandel, D. M. Perlman, B. H. Alexander, and P. D. Scanlon, "Estimates of restrictive ventilatory defect in the mining industry. considerations for epidemiological investigations: a cross-sectional study," BMJ Open, vol. 3, no. 7, Article ID e002561, 2013.

[19] F. R. Wouters, "EPIX: an interactive computer application program for cross-sectional epidemiological, periodontal investigations from X-ray films," Computer Methods and Programs in Biomedicine, vol. 26, no. 2, pp. 183-192, 1988.

[20] M. Ortega, M. Almela, A. Soriano et al., "Bloodstream infections among human immunodeficiency virus-infected adult 
patients: epidemiology and risk factors for mortality," European Journal of Clinical Microbiology and Infectious Diseases, vol. 27, no. 10, pp. 969-976, 2008.

[21] S. R. Ferrer, A. Strina, S. R. Jesus et al., "A hierarchical model for studying risk factors for childhood diarrhoea: a case-control study in a middle-income country," International Journal of Epidemiology, vol. 37, no. 4, pp. 805-815, 2008. 


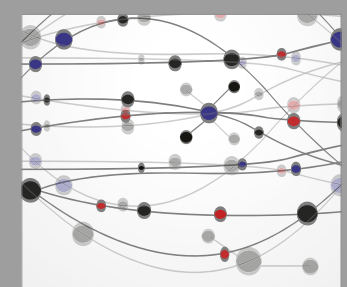

The Scientific World Journal
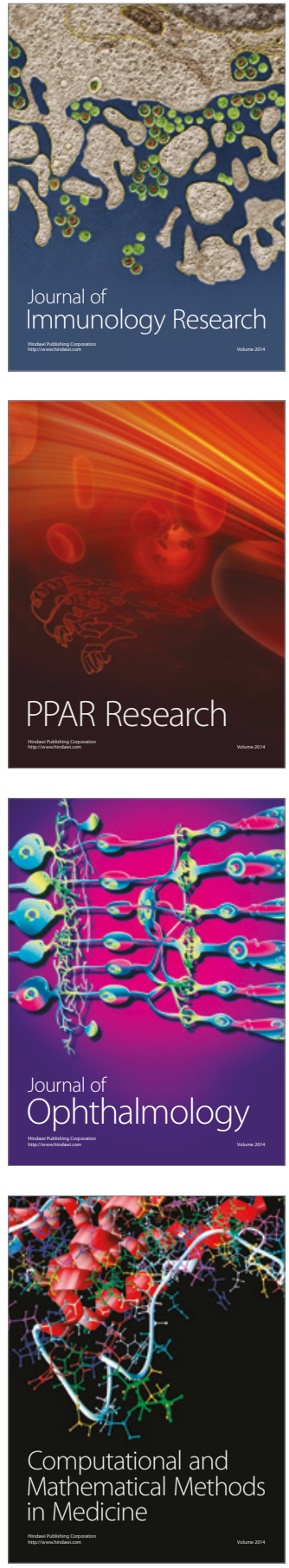

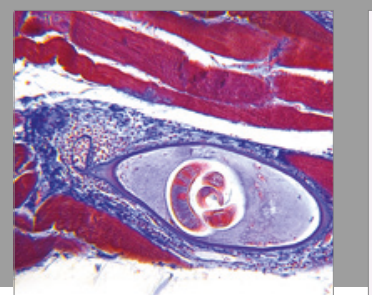

Gastroenterology Research and Practice
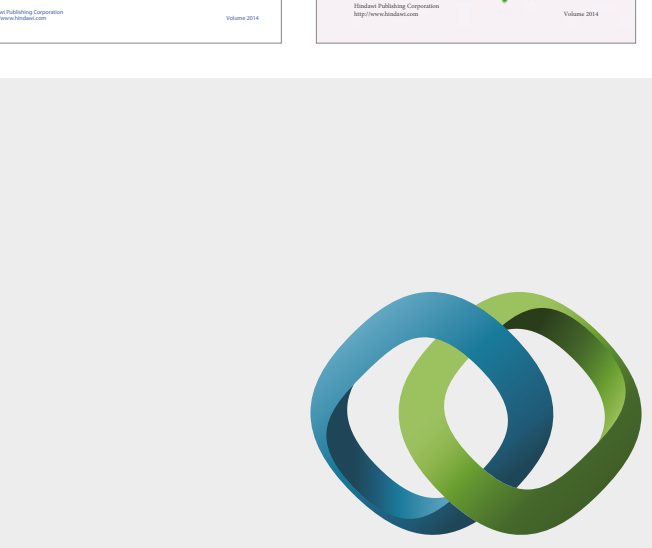

\section{Hindawi}

Submit your manuscripts at

https://www.hindawi.com
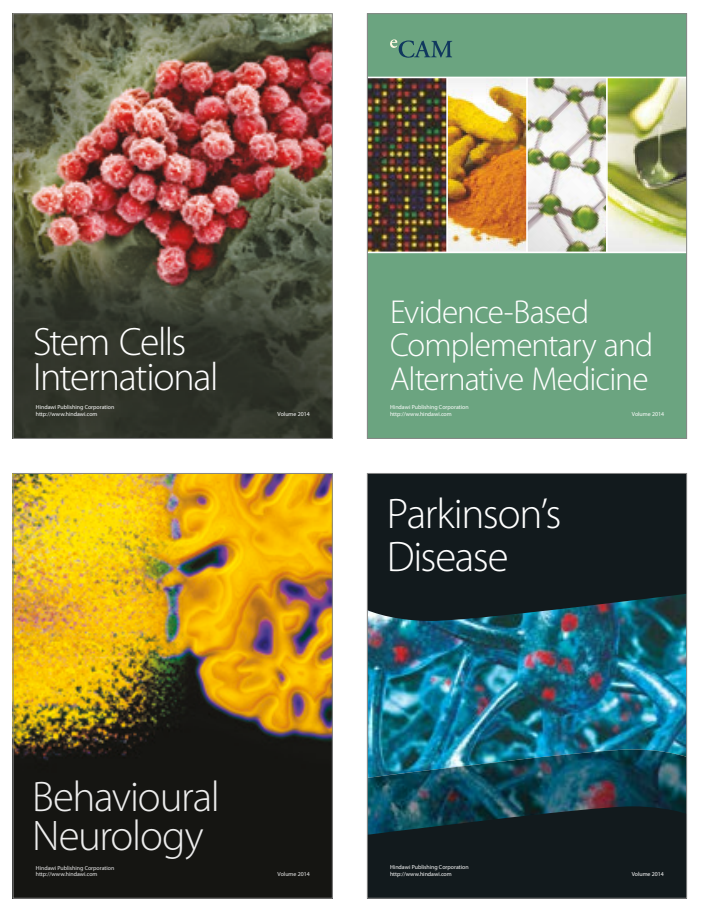
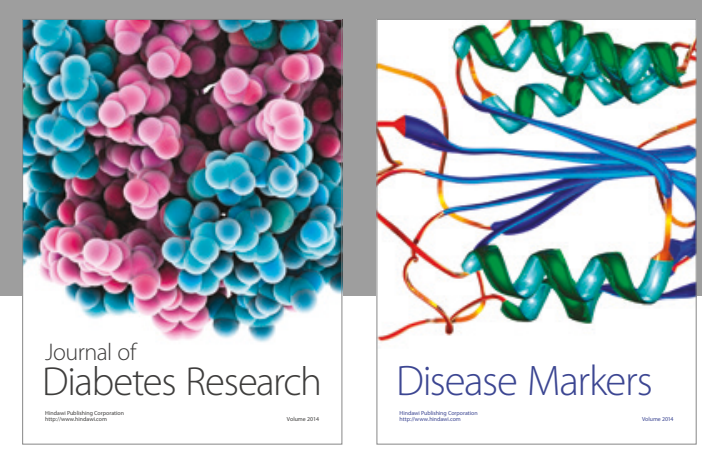

Disease Markers
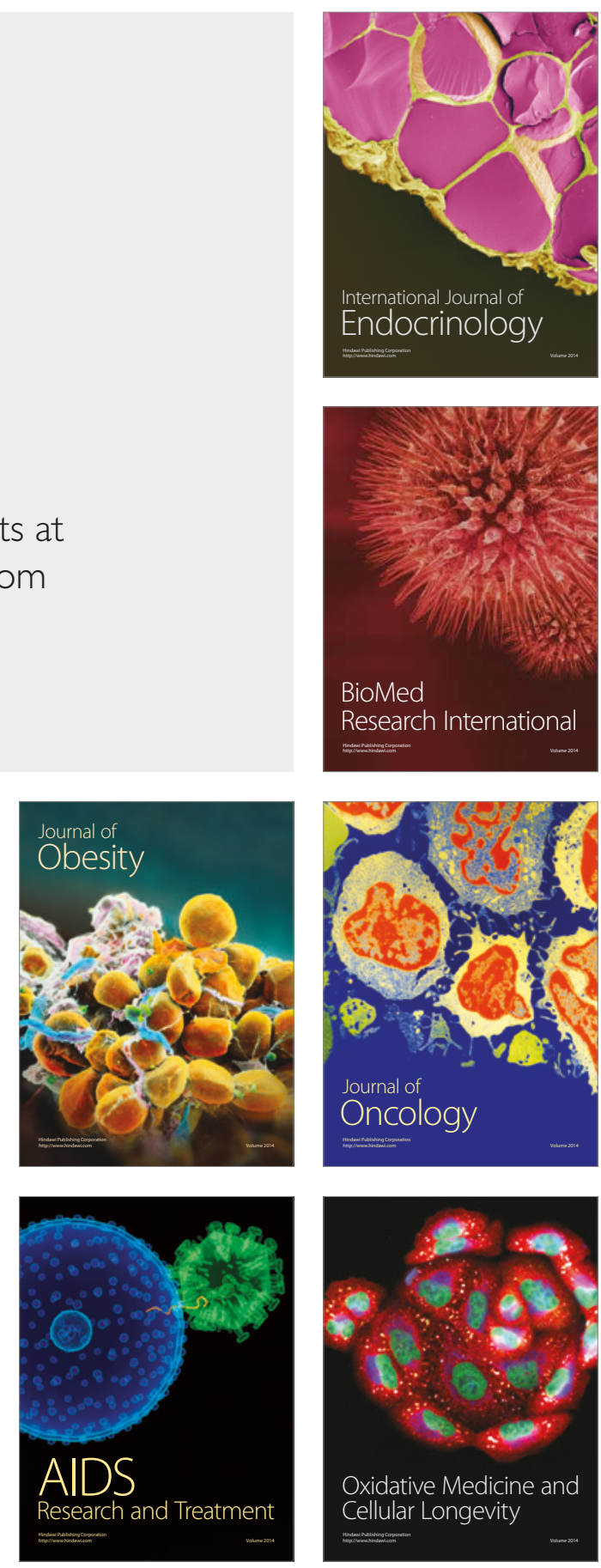